\title{
Quantifying the 3-Dimensional Shape of Lunar Regolith Particles Using X-Ray Computed Tomography and Scanning Electron Microscopy at Sub- $\lambda$ Resolution
}

\author{
Ann N. Chiaramonti, ${ }^{1}$ Jay D. Goguen, ${ }^{2}$ and Edward J. Garboczi ${ }^{1}$ \\ 1. Material Measurement Laboratory, National Institute of Standards and Technology, Boulder USA. \\ 2. Jet Propulsion Laboratory, California Institute of Technology, Pasadena USA.
}

Impacts are the dominant process shaping the regoliths of most airless solar system bodies. The impact process that controls the particle size and shape distribution of, for example, lunar soils is both destructive (comminution) and constructive (agglutinization) and is very different from processes that form terrestrial soils or result from grinding analogs in a mortar and pestle. Describing particle shapes as 'irregular' is only a qualitative description, and there are an infinite number of quantitatively different shapes that are irregular.

We now know that, in addition to the size distribution, the specific shapes and orientations of regolith particles play a major role in determining the light scattering properties of lunar soil and other planetary regoliths [1,2]. Our goal is to model light scattering by using the full 3D shape (and size distribution) of actual lunar soil particles that are on loan for this study through NASA's Curation and Analysis Planning Team for Extraterrestrial Materials. By determining what aspects of the actual irregular particle shapes of lunar regolith influence what components of the scattering matrix for a volume of randomly oriented particles, we seek to advance the ability to quantitatively interpret spacecraft images, photometry, polarimetry, and spectral data.

The focus of this work is to determine the full 3-D shapes of actual Apollo regolith particles expressed using spherical harmonic ( $\mathrm{SH}$ ) coefficients up to order $\sim 20$, for a statistically relevant number of particles with size parameter down to $\mathrm{x}<2(\mathrm{x}=2 \pi \mathrm{r} / \lambda$; where $\mathrm{r}=$ particle radius and $\lambda$ is the wavelength of scattered light, $0.5 \mu \mathrm{m} \leq \lambda \leq 2.5 \mu \mathrm{m}$ ). These measurements will serve as a 'ground truth', and we believe this will be the first time such large numbers of actual measured lunar regolith 3D particle shapes measured with this high spatial resolution will be used as inputs to scattering models for interpretation of intensity, polarization, and spectra of scattered light. Of course, a fraction of the particles may not be wellrepresented by spherical harmonic coefficients [4]. Figure 1 shows examples of a non-SH particle (a), and an SH particle (b), both with the same volume equivalent spherical diameter (VESD, diameter of sphere with same volume as the particle).

The regolith samples used in this study [3] include an Apollo 11 mare soil (10084) and an Apollo 14 Fra Mauro highland soil (14163). Both are $<1 \mathrm{~mm}$ fractions, otherwise unsorted, and should be representative examples of the sizes, shapes, and compositions of the particle types that control scattered light. The soils were embedded in an epoxy matrix for X-ray computed tomography (XRCT) studies and suspended in ethanol and drop cast for scanning electron microscopy (SEM) analysis.

Example reconstructed images of real $37 \mu \mathrm{m}$ diameter particles from Apollo $11 \& 14$ are shown in Figure 1. An accurate $\mathrm{SH}$ model can be generated for particles $\sim 10$ voxels across [4]. For our lunar regolith, this corresponds to particles with a radius of $\sim 3.5 \mu \mathrm{m}$ using a $0.7 \mu \mathrm{m}^{3}$ voxel size, which we have found to be the practical lower bound in this work due to limits of the sample preparation process and the XRCT technique itself. Particles as small as $\mathrm{x} \sim 2$ (corresponding to a radius of $0.16 \mu \mathrm{m}$ for $\lambda=0.5 \mu \mathrm{m}$ ) can potentially contribute important features to the average scattering matrix of the bulk soils. To compensate for this lack of 3D XRCT data for these small sizes, we used SEM imaging (Figure 2) to compile a survey of $2 \mathrm{D}$ particle sizes and aspect ratios designed to overlap the $0.7 \mu \mathrm{m}$ XRCT lower limit and extend the 
size and shape estimates to 0.1 to $0.2 \mu \mathrm{m}$ radii. The SEM data will allow us to evaluate the premise that the smaller particles whose shapes cannot be measured with XRCT are self-similar in shape to the larger particles that can $[5,6]$.

\section{References:}

[1] Mishchenko, M.I. and L.D Travis. Appl. Optics 33 (1994) 7206.

[2] Kahnert, M. J. Quant. Spectrosc. Radiat. Transfer 123 (2013) 62.

[3] Lunar Soil Compendium. http://curator.jsc.nasa.gov/Lunar/lsc/.

[4] Garboczi, E.J. Powder Technology 207 (2011) 96.

[5] Garboczi, E.J., X. Liu and M.A. Taylor. Powder Technology 229 (2012) 84.

[6] This work is a contribution of the US Government and is not subject to copyright in the United States.

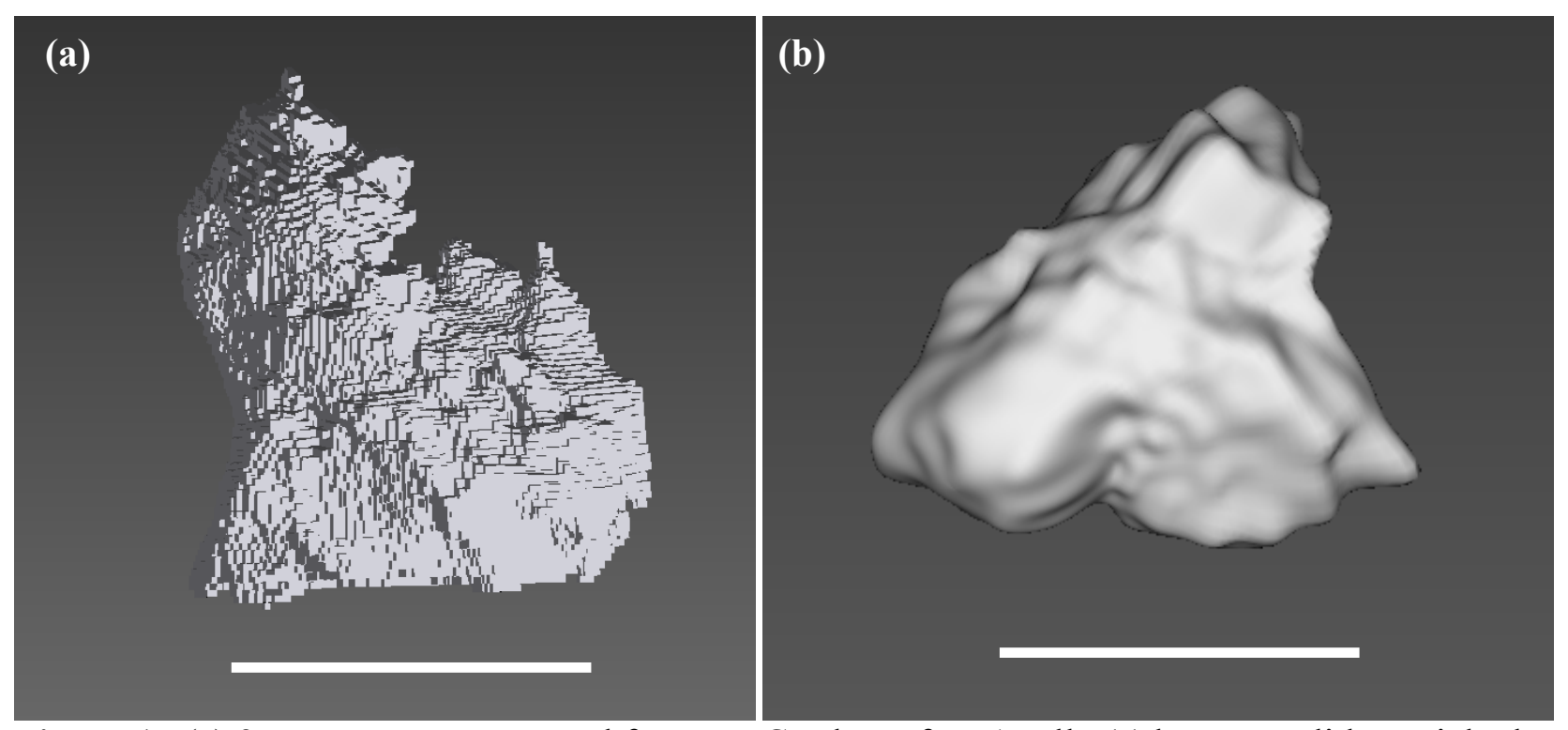

Figure 1. (a) 3D Image reconstructed from XRCT data of an Apollo 11 lunar regolith particle that is not well-represented using spherical harmonics, VESD $=37 \mu \mathrm{m}$. (b) Apollo 14 lunar regolith particle that is well-represented by spherical harmonics, VESD $=37 \mu \mathrm{m}$. The scale bar is $40 \mu \mathrm{m}$.
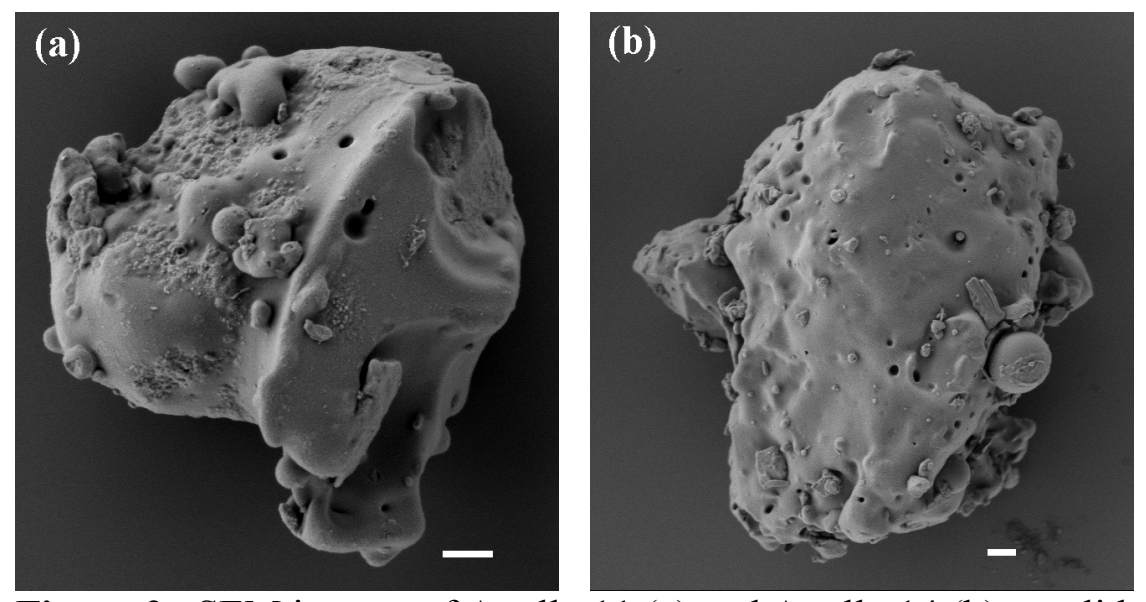

Figure 2. SEM images of Apollo 11 (a) and Apollo 14 (b) regolith particles. Scale bars are $400 \mathrm{~nm}$. 